\title{
AMILOIDOSIS VESICAL SECUNDARIA
}

\author{
J.L. PALMERO MARTÍ, A. BUDIA ALBA, S. ARLANDIS GUZMÁN, \\ A. BENEDICTO REDÓN, M. HERNÁNDEZ MARTÍ*, J.F. JIMÉNEZ CRUZ
}

Servicio de Urología. *Servicio de Anatomía Patológica. Hospital Universitario La Fe. Valencia.

Actas Urol Esp. 28 (3): 238-242, 2004

\section{RESUMEN}

\section{AMILOIDOSIS VESICAL SECUNDARIA}

La afectación vesical en la amiloidosis es un hecho poco frecuente, pudiendo distinguir entre formas primarias de amiloidosis vesical y formas de amiloidosis sistémica con afectación vesical (amiloidosis vesical secundaria), lo cual es importante para el urólogo. Ésta constituye una entidad patológica muy infrecuente, en base a la escasez de referencias en la literatura (estimamos que el número de casos de amiloidosis vesical secundaria comunicado no supera los 20). El caso que presentamos corresponde a una amiloidosis vesical secundaria en el seno de una artritis reumatoide de larga evolución, que debutó con hematuria severa. Señalamos la importancia que la inmunohistoquímia representa para el diagnóstico.

PALABRAS CLAVE: Amiloidosis vesical secundaria. Hematuria. Inmunohistoquímica.

\section{ABSTRACT}

\section{SECONDARY BLADDER AMYLOIDOSIS}

The bladder involvement is a very unusual fact in systemic amyloidosis. The distinction of primary and systemic amyloidosis disease with bladder involvement (secondary bladder amyloidosis) is important to the urologist. Secondary amyloidosis of the bladder is a rare disease entity (approximately 20 cases published). We document a case of a woman with a large history of rheumatoid arthritis who developed severe macrohematuria. Diagnosis was done by biopsy that revealed amyloidosis, and it was confirmed with an immunohistochemical staining of the specimens that defined the process as amyloid AA (secondary amyloidosis).

KEY WORDS: Scondary bladder amyloidosis. Hematuria. Inmunohistochemistry.

$\mathrm{L}^{\mathrm{a}}$ a amiloidosis se puede definir por el depósito extracelular de la proteína fibrilar amiloide en una o más localizaciones del organismo. Estos depósitos pueden producirse a distintos niveles del tracto genitourinario, estando descritas como hechos poco frecuentes en la literatura, la afectación de pelvis renal y uréter, vejiga, próstata, vesículas seminales, conductos deferentes, testículo, uretra y pene ${ }^{2}$.
Podemos clasificar las formas de amiloidosis del tracto genitourinario en dos grandes grupos: formas primarias o idiopáticas y formas secundarias a procesos inflamatorios crónicos o ciertas neoplasias ${ }^{4}$. La afectación vesical es un hecho poco habitual para ambas formas de amiloidosis, siendo mucho más infrecuentes las formas de amiloidosis vesical secundaria, que no superan los 20 casos publicados ${ }^{1-5,8}$, frente a los 160 de 
formas primarias ${ }^{10}$. Dentro de las formas secundarias, la artritis reumatoide constituye la enfermedad sistémica más frecuentemente asociada. Por otro lado, la distinción entre formas primarias y secundarias de afectación vesical comporta características clínico-patológicas distintas, como la naturaleza del material amiloide (AL en las formas primarias y AA en las secundarias) y la distinta localización de los depósitos de amiloide en la pared vesical, que conlleva una mayor frecuencia e intensidad de la hematuria en las amiloidosis secundarias, debido al depósito alrededor de los capilares de la submucosa que condiciona hematurias más severas y en ocasiones de difícil tratamiento, pudiendo ocasionar el fallecimiento del enfermo por la misma.

\section{CASO CLÍNICO}

Paciente mujer de 72 años, con antecedente de artritis reumatoide de larga evolución en tratamiento con AINES y en estudio por cuadro diarreico crónico de varios meses de evolución, por parte del Servicio de Digestología de otro centro. En el transcurso de una colonoscopia diagnóstica, la paciente sufre una perforación iatrogénica del colon descendente, motivo por el cual se remite de manera urgente a nuestro centro. Con el diagnóstico de perforación intestinal la enferma es intervenida de urgencia por el equipo de guardia de Cirugía General, realizando una laparotomía media con sutura primaria del colon descendente. Tras el sondaje intraoperatorio de la paciente, se produce la aparición inmediata de hematuria franca, motivo por el que se nos consulta. Valorada inicialmente la paciente y ante la ausencia de otra sintomatología asociada se decide manejo conservador y ver evolución. En las horas posteriores a la intervención se acrecentó la intensidad de la hematuria, instaurando un lavado vesical continuo y tratamiento intravenoso con E-aminocaproico, que no resolvió el cuadro produciéndose un cuadro de anemización progresiva que precisó la transfusión de seis unidades de concentrado de hematíes en los días posteriores. Posteriormente la intensidad de la hematuria remitió, aunque en las dos semanas posteriores a la intervención, se repitió en forma de episodios autolimitados que cedían parcial- mente con medidas conservadoras. A los seis días de la intervención se realizó ecografía que mostró ocupación vesical por una imagen que sugería coágulo y cistoscopia, que no fue concluyente por la ocupación intraluminal por un gran un coágulo. Durante la exploración, se realizó un intento de lavado que fue infructuoso por dolor, pudiendo tan sólo explorar el área trigonal, apreciando un coágulo filiforme que asomaba por el meato ureteral izquierdo. Se solicitaron pruebas de imagen: urografía intravenosa (Fig. 1a) que evidenció la normalidad de ambos sistemas ureteropielocaliciales, que aparecían discretamente ectásicos por la presencia de múltiples defectos de repleción intravesicales que sugerían presencia del coágulo ya conocido. La TAC (Fig. 1b) no aportó

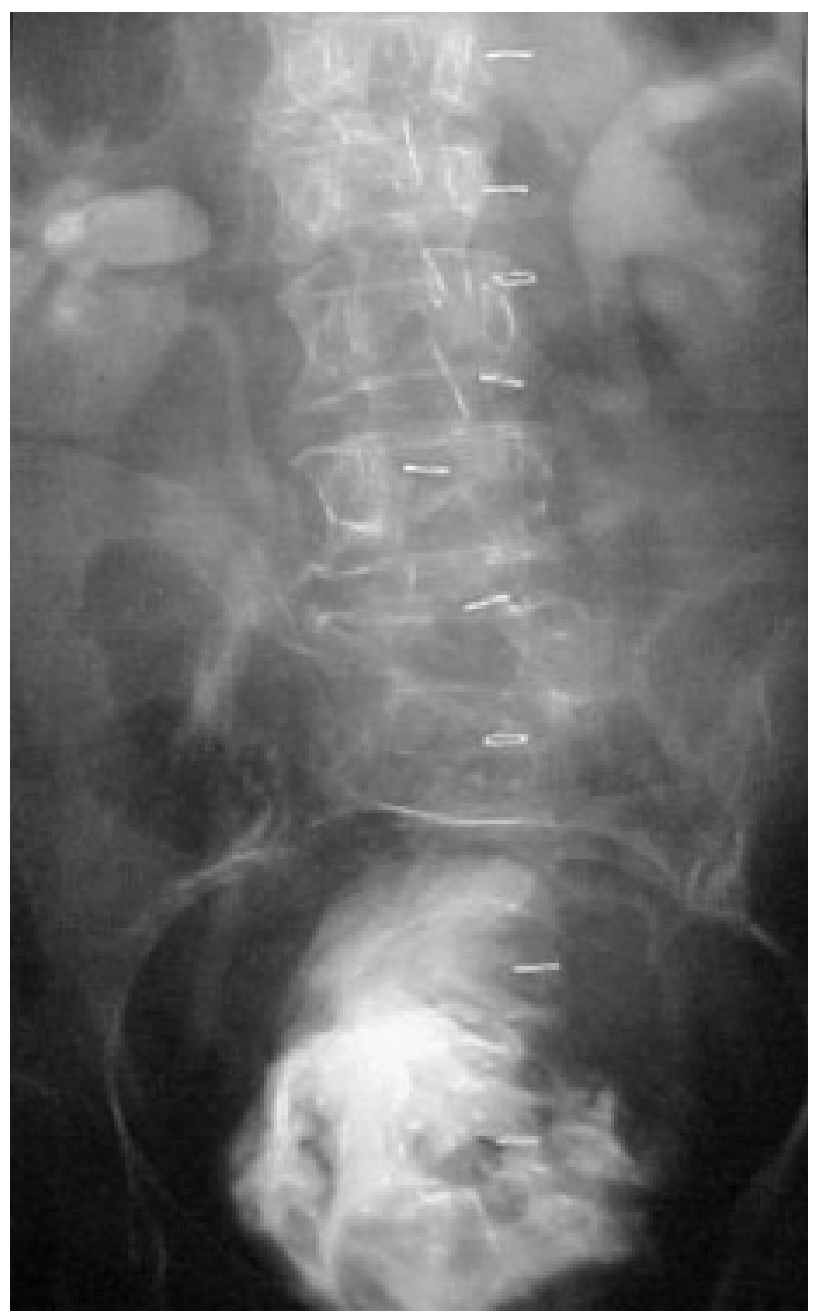

FIGURA 1a: Imagen de urografia intravenosa en la que se aprecian diversos defectos de repleción intravesicales por presencia de coágulos en el interior de la vejiga. 


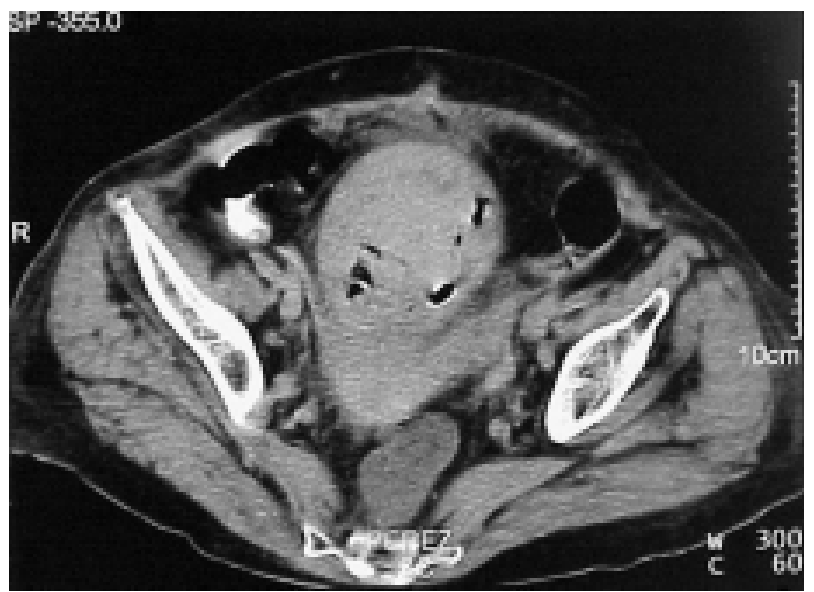

FIGURA 1b: Corte de TAC que muestra la ocupación vesical por coágulo organizado y presencia de burbujas de aire intravesical motivadas por intentos de extracción de aquel.

más información, con hallazgos similares a la exploración anterior, descartándose patología del tracto urinario superior.

Ante la persistencia de los episodios de hematuria y la emisión de coágulos se programó exploración bajo anestesia general, apreciando tras profuso lavado con Ellick, la presencia de una lesión excrecente y de aspecto aparentemente tumoral que se descolgaba desde cúpula por toda la pared posterior, respetando tan sólo el área trigonal-peritrigonal. Ante la sospecha de tumor vesical se realizó resección transuretral extensa dejando la lesión a plano. El estudio anatomo-patológico del material remitido evidenció la presencia de material amiloide que se distribuía alrededor de los vasos submucosos, como podemos apreciar en la tinción con hematoxilina-eosina (Fig. 2). El carácter eosinófilo de este material también fue puesto de manifiesto mediante tinción con rojo congo. Por otra parte, el estudio inmunohistoquímico del material, con anticuerpos monoclonales (clone mcl) específicos frente a la proteína AA del amiloide, confirmaron que los depósitos perivasculares submucosos correspondian a amiloide AA que se teñian de manera característica mediante reacción de inmunoperoxidasa (Fig. 3). Con todos estos hallazgos se emitió el diagnóstico de amiloidosis vesical secundaria.

La evolución post-operatoria de la paciente fue favorable siendo dada de alta al $10^{\circ}$ día tras la intervención y seguida posteriormente en

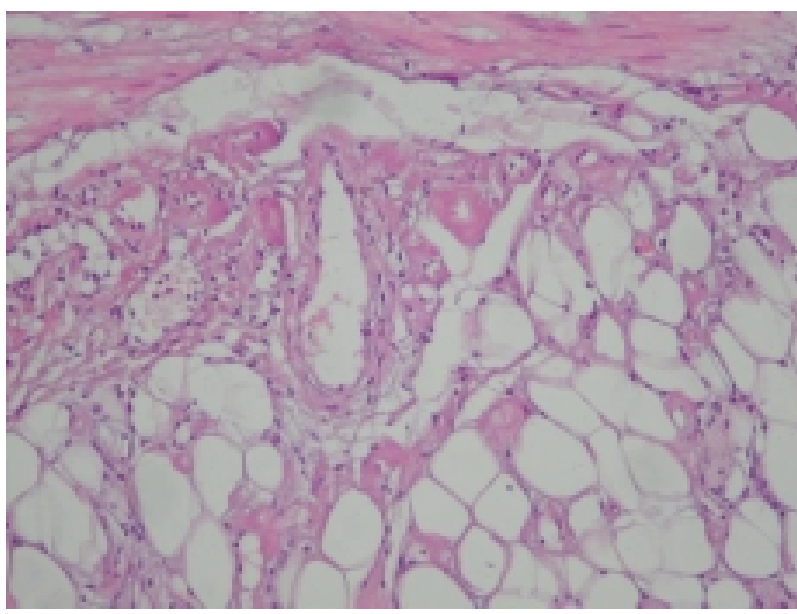

FIGURA 2: Tinción de Hematoxilina-Eosina que muestra depósitos de material eosinófilo (amiloide) alrededor de los vasos submucosos.

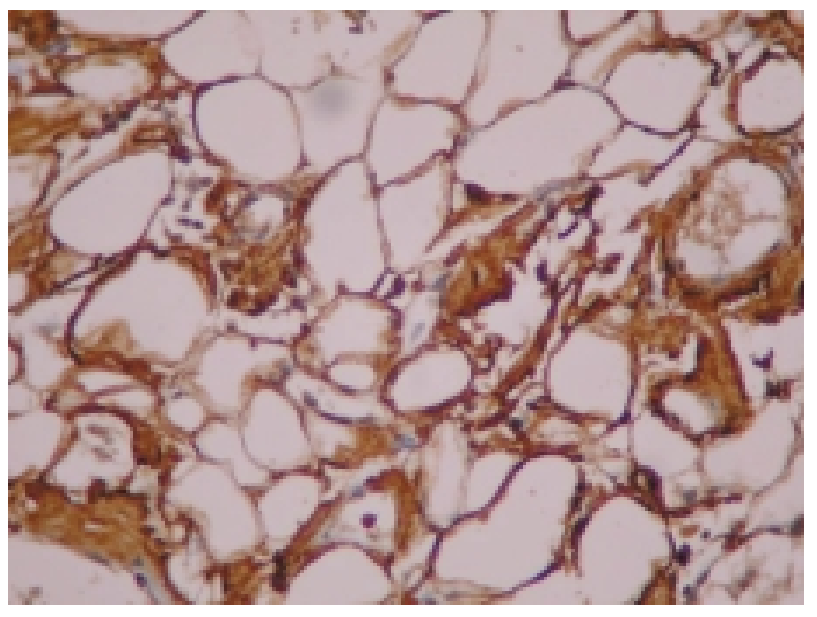

FIGURA 3: Depósitos de material amiloide alrededor de los vasos submucosos vesicales teñidos mediante la técnica de inmunoperoxidasa. La fijación de anticuerpos monoclonales Ab-1 (clone mc1) a las fibrillas de amiloide y posterior tinción de las mismas por reacción de la peroxidasa, permite tipificar estos depósitos como amiloide AA (amiloidosis secundaria).

nuestras consultas externas. Actualmente el seguimiento de la paciente es de 30 meses, realizando cistoscopias trimestrales durante el primer año, y semestrales durante el segundo, sin haberse producido hasta el momento ninguna recidiva de la enfermedad. Tan sólo se apreció en los primeros controles cistoscópicos, áreas focales de material amiloide en la mucosa que no han generado ninguna complicación posterior (Fig. 4). 


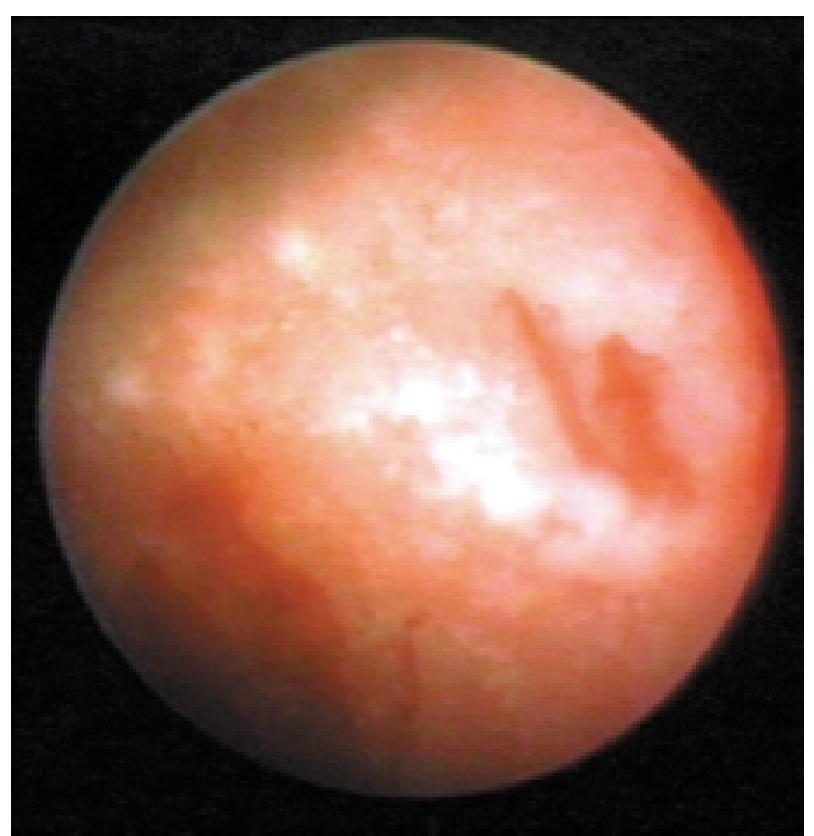

FIGURA 4: Imagen cistoscópica en control tras RTU (1 mes). Podemos apreciar pequeñas placas amarillentas de material amiloide que se elevan sobre la mucosa vesical.

\section{DISCUSION}

La amiloidosis fue descrita inicialmente por Rokitansky en 1842, y su nombre lo proporciona Virchow en 1883 al observar que el material de depósito presentaba características tintoriales similares al almidón ${ }^{2}$. Definida como el depósito extracelular de amiloide, la siguiente clasificación es útil para entenderla desde un punto de vista clínico: amiloidosis primaria o idiopática (tipo $\mathrm{AL}$ ), amiloidosis secundaria (tipo $\mathrm{AL}$ ) y otras formas de amiloidosis que incluyen formas heredofamiliares, las asociadas a mieloma múltiple, formas seniles de amiloidosis y formas de amilodosis focales que afectan a distintos órganos simulando tumores ${ }^{2,9}$.

La primera descripción de amiloidosis vesical la proporciona Solomin en 1887 y Corbitt et al. aportan en 1944 la primera imagen endoscópica de esta enfermedad. La afectación vesical sintomática en el seno de enfermedades sistémicas (formas secundarias) es muy infrecuente, aportando Bender y Kelly en 1969 el primer caso de amiloidosis vesical secundaria en un paciente con artritis reumatoide ${ }^{2}$. La enfermedad sistémica más frecuentemente asociada a esta entidad es la artritis reumatoide como en el caso que aportamos. La serie más amplia de esta entidad corresponde a Nurmi et al. con $5 \operatorname{casos}^{2,5}$, estimando que el número de casos sintomáticos de esta entidad que se han comunicado no supera $\operatorname{los} 20^{1-5,8}$. Señalar que a pesar de su infrecuencia, la afectación vesical es más frecuente de lo que conocemos, ya que ésta no suele manifestarse clínicamente y correspondería al estudio microscópico de necropsias de pacientes con amiloidosis sistémicas ${ }^{3,7}$. A pesar de ello, y en contra de lo que pueda parecer, la afectación vesical en el seno de las amiloidosis sistémicas se ha descrito en un porcentaje muy inferior, respecto al de las amiloidosis vesicales primarias ${ }^{2-4,6}$.

El síntoma de presentación más frecuente es la hematuria ${ }^{2-4,6,9,11-14}$, que como en el caso que nosotros aportamos suele ser de intensidad importante y en ocasiones de difícil control. En la bibliografia revisada, la hematuria se presenta con mayor incidencia en los pacientes con amiloidosis vesicales secundarias. Este hecho lo explican varios autores por el depósito de material amiloide alrededor de los capilares de la submucosa, hecho que dificulta la vasoconstricción hemostática de los mismos en respuesta a la hemorragia ${ }^{2-4,6,7}$. El factor desencadenante de la hematuria no es del todo conocido aunque diversos autores apuntan a la irritación de la mucosa vesical por diversos factores como la infección urinaria ${ }^{3}$ o instrumentaciones endoscópicas ${ }^{4,6}$ y/o el mismo sondaje ${ }^{6}$, como en el caso que presentamos.

En la amiloidosis vesical la imagen endoscópica es inespecífica ${ }^{9,10,13}$ pudiendo encontrar diversos hallazgos en esta exploración, como zonas hemorrágicas con áreas de edema alrededor que dan aspecto de proceso inflamatorio ulcerado o bien adquirir morfología de lesiones pseudotumorales excrecentes (nuestra paciente) y que simulan un tumor sólido ${ }^{9}$. En otras ocasiones, la cistoscopia puede mostrar placas blanco-amarillentas sobreelevadas ${ }^{9,11,12}$, que corresponden a los depósitos de material amiloide en la pared (Fig. 4) $\mathrm{y}$ que pueden hacernos pensar en esta entidad. No obstante, el diagnóstico definitivo de una amiloidosis vesical nos lo proporcionará el estudio histopatológico ${ }^{2-9,11-15}$, demostrando la presencia de material amiloide mediante diversas técnicas (Hematoxilina-Eosina, Rojo Congo). De otra parte, la importancia de las técnicas de inmu- 
nohistoquímia en el diagnóstico, se debe a su capacidad de distinguir entre formas de amiloide AL y AA, lo cual permite hablar de formas de amiloidosis primarias (amiloide AL) y secundarias (AA). En nuestro caso, el empleo del anticuerpo monoclonal Ab-1 (clone mc1) y posterior tinción mediante inmunoperoxidasa, nos permitió el diagnóstico de amiloidosis vesical secundaria, en base a la especificidad de éstos para el amiloide AA y el mayor rendimiento diagnóstico que permite la asociación de estas técnicas al Rojo Congo ${ }^{16-18}$.

En lo que respecta al tratamiento, en los casos sintomáticos se centra en el control de la hematuria, mediante tratamiento conservador inicial y en caso de persistencia o gran cuantía es posible emplear diversos procedimientos que van desde la instilación de $\mathrm{DMSO}^{5}$, resección transuretral hemostática ${ }^{1-4,6,7}$, ligadura de arterias hipogástri$\operatorname{cas}^{3,4,7}$, incluso cistectomía en casos extre$\operatorname{mos}^{1,3,6,8}$.

Es necesario un seguimiento estrecho de estos enfermos por la posibilidad de recurrencia de la enfermedad y descartar en la coexistencia de un carcinoma vesical ${ }^{2}$.

\section{CONCLUSIONES}

Se debe sospechar la posibilidad de amiloidosis vesical secundaria en aquellos casos en los que aparezca una hematuria severa en el contexto de pacientes con procesos inflamatorios y/o infecciosos crónicos. Dada la inespecificidad del cuadro clínico y de los hallazgos de la exploración cistoscópica (aunque ésta en ocasiones puede hacernos sospechar la enfermedad), el diagnóstico definitivo lo establecerá el estudio histopatológico e inmunohistoquímico del material de biopsia. Consideramos necesario el seguimiento periódico de estos pacientes, mediante cistoscopias periódicas, ante la posibilidad de recurrencia de la enfermedad, como se ha descrito en la literatura revisada.

\section{REFERENCIAS}

1. CABALLERO GINÉ JM, BORRAT FONT P, MARTÍ PICAS L.: Amiloidosis vesical: presentación de dos casos. Actas Urol Esp 2000; 24; 413-415.

2. BEGARA MORILLAS F, SILMI MOYANO A, ESPINOSA FERNÁNDEZ B.: Amiloidosis vesical en una paciente con sospecha de carcinoma vesical. Arch Esp Urol 1994; 47: 176-179.
3. TESEDO CUBERO J, RISTOL RM, FERNÁNDEZ ZUAZU J.: Hematuria masiva y perforación vesical, complicación de una amiloidosis sistémica. Arch Esp Urol 1991; 44: 862-864.

4. BIELSA O, ROMERO JA, ALCARAZ A.: Amiloidosis vesical secundaria. Una rara causa de hematuria. Actas Urol Esp 1990; 14: 76-77.

5. NURMI MJ, EKFORS TO, RAJALA PO.: Intravesical dimethyl sulfoxide instillations in the treatment of secondary amyloidosis of the bladder. J Urol 1990; 143: 808-809.

6. SANZ JAKA JP, ARRINDA YEREGUI JM, MENDIBIL DAKAL J.: Afectación vesical en la amiloidosis sistémica. Arch Esp Urol 1988; 41: 325-328.

7. NURMI MJ, EKFORS TO, PUNTALA PV.: Secondary amyloidosis of the bladder: a cause of massive hematuria. $J$ Urol 1987; 138: 44-45.

8. LEIVA GALVIS O, RESEL ESTÉVEZ L.: Patologías excepcionales en Urología: hematuria incoercible por amiloidosis vesical. Luzán 5, S.A. 2002: 85-95.

9. VICENTE J, ALGABA F.: Semiología diagnóstica endovesical: amiloidosis vesical. Pulso Ediciones S.A. 1998: 105-113.

10. MALEK RS, WAHNER-ROEDLER DL, GERTZ MA. Primary localized amiloidosis of the bladder: experience with dimethyl sulfoxide therapy. J Urol 2002; 168: 1018-1020.

11. QUINTANA DE LA ROSA JL, RUBIAL MOLDES M, PESQUEIRA SANTIAGO D.: Amiloidosis vesical primaria. Actas Urol Esp 2001; 25: 530-532.

12. AUGE BK, HALUSZKA MM.: Primary amyloidosis of the bladder. J Urol 2000; 163: 1867-1868.

13. GONZÁLEZ NARANJO F, VELASCO ALONSO J, MÉNDEZ LÓPEZ M.: Amiloidosis vesical. Actas Urol Esp 1992; 16: 579-581.

14. LÓPEZ LÓPEZ C, ROMERO MAROTO J, MAYOL BEDTA MJ.: Amiloidosis primaria de localización vesical: actualización diagnóstica y terapéutica. Arch Esp Urol 1993; 46: 864-867.

15. EHARA H, DEGUCHI T, YANAGIHARA M.: Primary localized amiloidosis of the bladder: an inmunohistochemical study of a case. J Urol 1992; 147: 458459.

16. LINKE RP, GARTNER HV, MICHELS H.: High-sensitivity diagnosis of AA amyloidosis using Congo red and immunohistochemistry detects missed amyloid deposits. J Histochem Cytochem 1995 sep; 43: 863-869.

17. LINKE RP.: Identification of amyloid protein AA with monoclonal antibody. Blut 1982; 45: 407-409.

18. LINKE RP.: Identification of AA-type amyloid in tissue sections using monoclonal antibodies. In Peeters H, ed. Protides Biol Fluids Oxford. Pergamon Press 1983; 31: 835-838.

Dr. J.L. Palmero Martí

Servicio de Urología

Hospital Universitario La Fe

Avda. Campanar, 21

46009 Valencia

(Trabajo recibido el 23 junio de 2003) 